\title{
CFD ANALYSIS OF HEAVY LIQUID METAL FLOW IN THE CORE OF THE HELIOS LOOP
}

\author{
A. BATTA ${ }^{1 *}$, JAE HYUN CHO ${ }^{2}$, A.G. CLASS ${ }^{1}$, and IL SOON HWANG ${ }^{2}$ \\ ${ }^{1}$ Karlsruhe Institute of Technology/Institute for Nuclear and Energy Technologies (KIT/IKET) \\ P.O. Box 3640·D-76021 Karlsruhe Germany \\ ${ }^{2}$ Nuclear Transmutation Energy Research Center of Korea (NUTRECK) \\ Seoul National University, Daehak-dong, Gwanak-ku, Seoul, 151-744, South Korea \\ ${ }^{*}$ Corresponding author. E-mail : Abdalla.Batta@kit.edu
}

Received May 24, 2010

Accepted for Publication October 18, 2010

Lead-alloys are very attractive nuclear coolants due to their thermo-hydraulic, chemical, and neutronic properties. By utilizing the HELIOS (Heavy Eutectic liquid metal Loop for Integral test of Operability and Safety of PEACER ${ }^{2}$ ) facility, a thermal hydraulic benchmarking study has been conducted for the prediction of pressure loss in lead-alloy cooled advanced nuclear energy systems (LACANES). The loop has several complex components that cannot be readily characterized with available pressure loss correlations. Among these components is the core, composed of a vessel, a barrel, heaters separated by complex spacers, and the plenum. Due to the complex shape of the core, its pressure loss is comparable to that of the rest of the loop. Detailed CFD simulations employing different CFD codes are used to determine the pressure loss, and it is found that the spacers contribute to nearly 90 percent of the total pressure loss. In the system codes, spacers are usually accounted for; however, due to the lack of correlations for the exact spacer geometry, the accuracy of models relies strongly on assumptions used for modeling spacers. CFD can be used to determine an appropriate correlation. However, application of CFD also requires careful choice of turbulence models and numerical meshes, which are selected based on extensive experience with liquid metal flow simulations for the KALLA lab. In this paper consistent results of CFX and Star-CD are obtained and compared to measured data. Measured data of the pressure loss of the core are obtained with a differential pressure transducer located between the core inlet and outlet at a flow rate of $13.57 \mathrm{~kg} / \mathrm{s}$.

KEYWORDS : Pressure Loss, CFD, CFX, Star-CD, LACANES, HELIOS, PEACER

\section{INTRODUCTION}

Lead alloys are considered very attractive nuclear coolants due to their thermo-hydraulic, chemical, and neutronic properties. In the past, heavy liquid metals were employed in Russian submarines and they are now being addressed in research globally, with much research focusing on accelerator driven transmutation systems and lead or lead-alloy cooled fast reactors (LFR). Benchmarking for the OECD/NEA project LACANES (Lead Alloy Cooled Advanced Nuclear Energy Systems) aims at evaluating system code for characterization of the thermo-hydraulics of heavy-liquid metal cooled loops under forced and natural convection [1].

HELIOS (Heavy Eutectic liquid metal Loop for Integral test of Operability and Safety of PEACER), located in Seoul National University in the Republic of Korea, employs LBE (Lead Bismuth Eutectic) coolant.
The loop, shown in Figure 1, consists of a LBE pump that pumps the flow through an electrically heated mockup

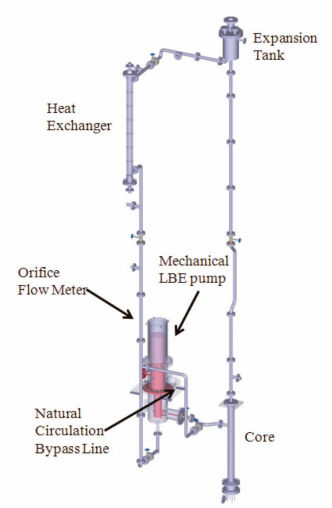

Fig. 1. HELIOS Loop, Showing Main Loop Components 
core into an expansion tank positioned approximately eight meters above the core. In the backflow, a LBE/oil heat exchanger cools the flow before it returns to the pump. The loop provides a bypass to the pump for natural convection experiments. A material test bypass is also available. The loop is equipped with various differential pressure sensors, which are located in the core, orifice flow meter, and gate valve, and thermocouples to characterize the system code behavior are also installed [2-4].

The results of system code modeling of the LACANES benchmarking phase I are summarized in Figure 2. This figure shows the accumulated pressure losses along the

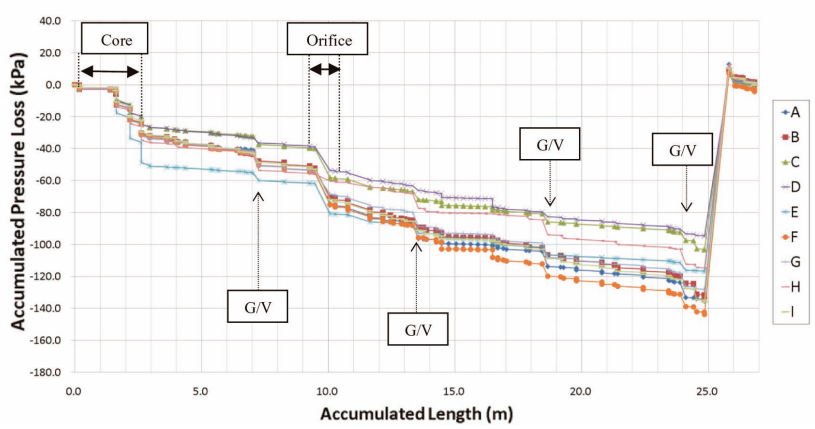

Fig. 2. Results of LACANES Benchmarking Phase-I by Using Handbook Correlation in High Mass Flow Rate Condition $(13.57 \mathrm{~kg} / \mathrm{s})$ length in the flow circuit, where the results were obtained by the contributions of nine organizations. As seen here, most of the participants computed similar pressure loss profiles along the loop. However, there is substantial scattering at the gate valves, the orifice flow meter, and in the mockup core. It is thus concluded that the complex geometry of the gate valves and also of the orifice flow meter necessitate the use of calibrated pressure loss correlations for these components. The predicted pressure loss of the mockup core shows inacceptable variations. Since the mockup-core is a vital reactor component, such a component should be predicted with reasonable confidence.

In this paper, numerical simulations have been carried out and the obtained results have been compared with the measured data of the core region in the HELIOS facility. The Star-CD and CFX code have been utilized.

\section{DESCRIPTION OF THE CORE REGION IN HELIOS FACILITY}

Figure 3 shows the flow pathline and detail drawings of the core region. The core consists of a long vessel with a concentric barrel containing a heating rod bundle in its center. The flow enters the mockup core vessel at approximately $2 / 3$ height through the vessel inlet. Next, it flows downwards in a gap between the vessel and the barrel (downcomer) to the lower plenum, where the flow direction is inverted. Within the barrel, the upward flow is heated by a four rod bundle [4].

The geometric data and Reynolds number for the tested flow rate $(13.57 \mathrm{~kg} / \mathrm{s})$ are summarized in Table 1.

Table 1. Geometric Data and Reynolds Number for Tested Flow Rate (13.57kg/s)

\begin{tabular}{c|c|c|c|c|c}
\hline \multirow{2}{*}{ Section } & Area & Length & Hydraulic Diameter & Average Velocity & \multirow{2}{*}{ Reynolds Number } \\
\cline { 2 - 5 } & $\mathrm{m}^{2}$ & $\mathrm{~m}$ & $\mathrm{~m} / \mathrm{s}$ & 0.68 \\
\hline Inlet pipe & 0.0019 & 0.16 & 0.049 & 0.13 & 0.92 \\
\hline Downcomer & 0.0097 & 1.19 & 0.018 & $1.65 \cdot 10^{5}$ \\
\hline Rod bundle & 0.0014 & 1.3 & 0.008 & $8.21 \cdot 10^{4}$ \\
\hline $\begin{array}{c}\text { Spacers } \\
\text { (3ea) }\end{array}$ & 0.0007 & 0.005 & 0.064 & $7.29 \cdot 10^{4}$ \\
\hline Outlet pipe & 0.0019 & 0.68 & 0.049 & 0.68 & $1.65 \cdot 10^{5}$ \\
\hline
\end{tabular}


Area change ratio of the rod bundle to spacers is about two, which means the ratio of Reynolds number is roughly a square root of two in a simple geometric condition. However, the rod bundle and spacers have Reynolds numbers of $8.21 \cdot 10^{4}$ and $7.29 \cdot 10^{4}$, respectively, because the hydraulic diameter of the spacers is larger than that of the rod bundle, which is derived from the smaller wetted perimeter of the spacers.

\section{NUMERICAL STUDY}

A closer inspection of the predicted pressure losses of the core shows that most of the pressure losses can be attributed to changes in the flow area. In particular, the various participants of the benchmarking project estimated the effects of the spacers quite differently. One reason for this scattering is the absence of a specific and unique design for the spacers, and thus the system code models for the spacers are tuned for specific spacer arrangements. Among the correlations for the spacers is the Rehme correlation [5-6] developed by KIT/IKET (Karlsruhe Institute of Technology/Institute for Nuclear and Energy Technologies) and used in the system code model applied by KIT/IKET. In order to improve predictions of the spacer pressure losses, detailed CFD simulations of the core were conducted. The CFD simulations were independently performed at KIT/IKET of Germany and NUTRECK (Nuclear Transmutation Energy Research Center of Korea) of Korea, and independent commercial CFD codes were employed. Specifically, Star-CD was applied at KIT/IKET and CFX at NUTRECK. Results were compared to the Rehme correlation, which was previously applied by KIT/IKET.

\subsection{Hydrodynamic Models}

In order to produce reliable predictions of the pressure losses in the mock-up core, two independent 3-dimensional CFD simulations employing similar models were performed. The core consists of four rods forming a single subchannel bounded by rods in its center, and the gap between the barrel and the rods forms additional subchannels, as shown in Figure 3. The system-code predictions indicate that pressure losses across the spacers dominate the overall pressure loss. The length of the core $(2.1 \mathrm{~m})$ suggests that flow development is a non-negligible effect, because the flow of most regions has a developing flow condition, not a fully developed flow condition. The complex core geometry requires simulation of all geometrical details of the mockup core. These considerations led to the choice of a high Reynolds number k- $\varepsilon$ turbulence model, i.e. the use of wall functions, for this first study, where the grid requirements are achievable at reasonable computational cost. The chosen turbulence model [7] is well suited to compute the developing flow and pressure losses due to obstructions, and is capable of predicting the essential features of the flow in the mockup core. Effects such as secondary flow that require more sophisticated turbulence models, i.e. nonlinear models, are of minor importance for the global pressure loss here. The convergence criterion of the numerical solution was chosen as $10^{-3}$ for RMS mass and momentum in the CFX simulation and Star-CD. In [8] an extensive study of a bundle flow with spacers is conducted and the results are compared to those of an accompanying water experiment. This study shows that (i) the Rehme correlation [5] applies for bundles with high number of rods; (ii) $\mathrm{k}-\varepsilon$ simulations where spacer pressure losses are dominant are accurate; and (iii) the mesh density in the spacer region is the most important factor for accurate simulation [8]. Furthermore, in [9], LBE rod bundle experiments performed at the KALLA Lab at KIT are compared to the Rehme correlation and show fluid independence. In the present study, only four fuel pin simulators are installed in the mockup core; consequently, the Rehme correlation is not expected to hold, but the previous numerical method is expected to accurately simulate the flow.

\subsection{Meshing}

The two independent simulations use the same turbulence model and fluid properties but different meshes. In the Star-CD simulation, a polyhedral mesh with 410,000 polyhedrals is used. Two prismatic wall layers yield a fine near wall mesh. The mesh density and structure were selected in accordance with our previous study in [8]. In the CFX simulation, a tetrahedral mesh with 2,620,000 tetrahedrals with refinement towards the walls is used. It uses 490,000 computational nodes. It should be noted that each polyhedral corresponds to a computational node, and hence the two simulations have similar overall

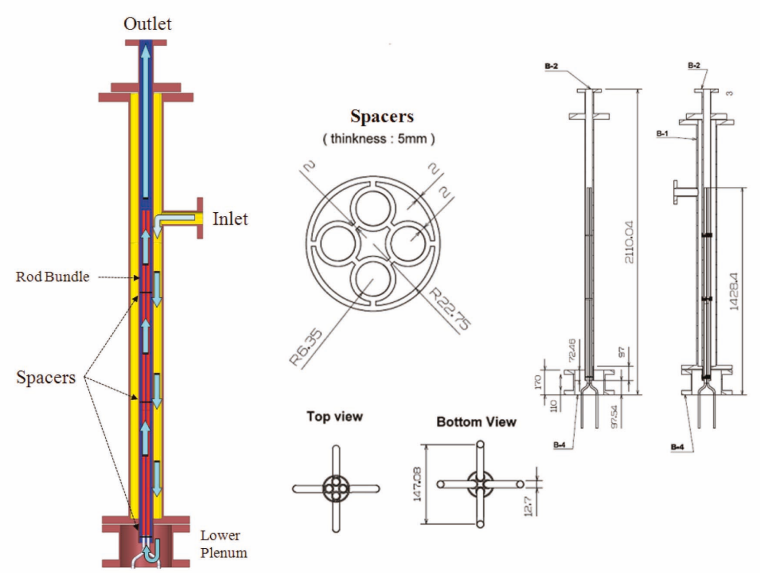

Fig. 3. Flow Pathline and Detail Drawings of the Core Region 
resolution. Figure 4 depicts a cross section in the computational domain showing the mesh in the lower plenum and the lower part of the core rods including the lower spacer.

\subsection{Boundary Conditions}

For the boundary conditions for the CFD models, the inlet, outlet, and walls are defined. At the inlet, the mass flow rate of the fluid was fixed and the direction of the flow was defined normal to the boundary. At the outlet, the average area pressure was applied as $0 \mathrm{~Pa}$. At all walls, a no-slip boundary condition was imposed. There is no buoyancy effect or thermal energy calculation in the CFD models because the experimental environmental conditions constitute an isothermal steady state $\left(250^{\circ} \mathrm{C}\right)$.

\subsection{Wall Treatment}

For a high Reynolds number $\mathrm{k}-\varepsilon$ turbulence model simulation, the proper choice of $y+$ values for the first

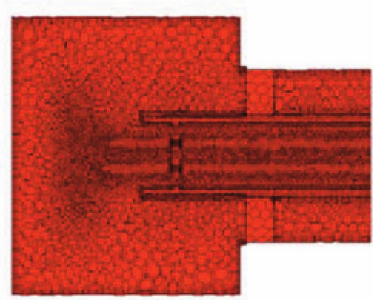

(a)

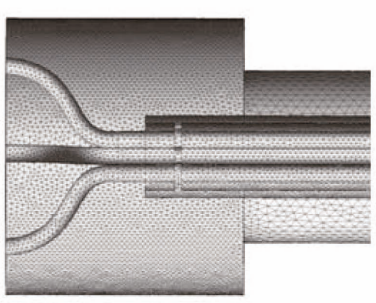

(b)
Fig. 4. Cross Section in the Computational Domain Showing Mesh in the Lower Plenum and the Lower Part of the Core Rods Including the Lower Spacer (a) Star-CD, (b) CFX

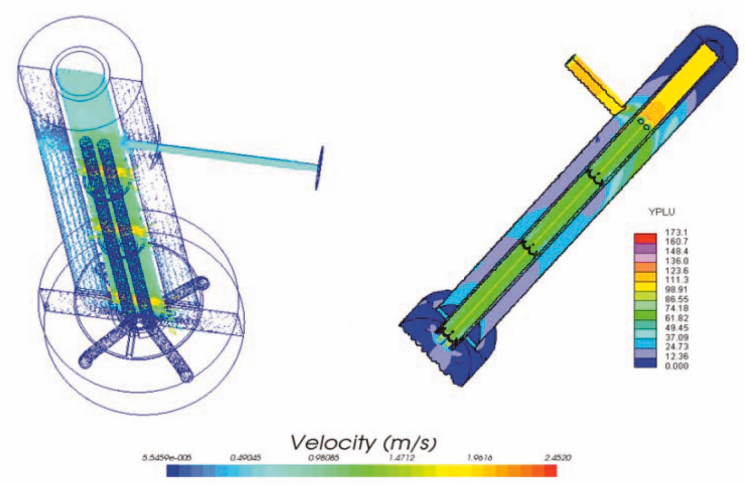

Fig. 5. Velocity Counters at a Plane Cross the Core Center and Resulting y+ Values for Flow Rate of $13.57 \mathrm{~kg} / \mathrm{s}$, Results of STAR-CD Code layer of cells is the most important factor and the value must not fall under 12. A small number satisfying this criterion is desirable for future temperature field simulations. The use of a high Reynolds number formulation for a rod bundle flow with spacers was verified in [8]. In the vicinity of the spacers, the formation of thin boundary layers requires dense meshes in the spacer region. Two layers of wall elements with a small wall distance are implemented. Figure 5 and Figure 6 show that proper $y+$ values have been selected in both the CFX and Star-CD simulations, respectively.

\subsection{Results}

Resulting pressure fields are shown in Figure 7 and Figure 8 . The most important feature of the pressure field

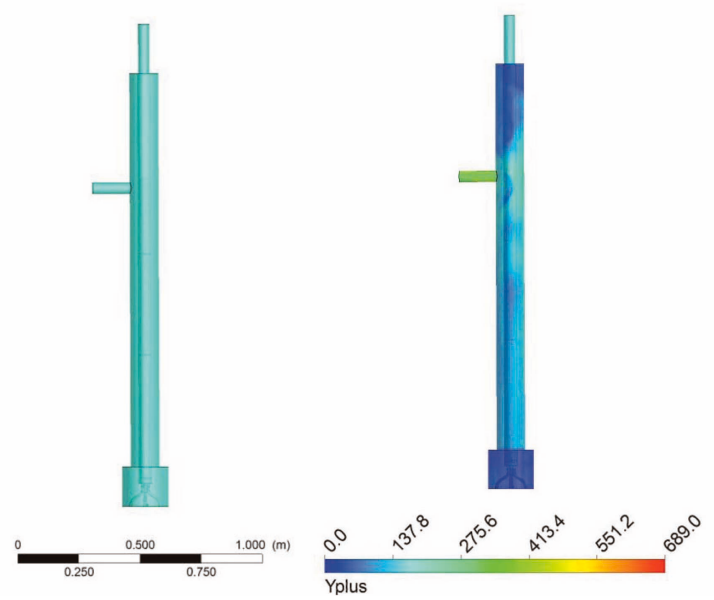

Fig. 6. Computational Domain and Resulting y+ Values for Flow Rate of $13.57 \mathrm{~kg} / \mathrm{s}$, Results of CFX Code
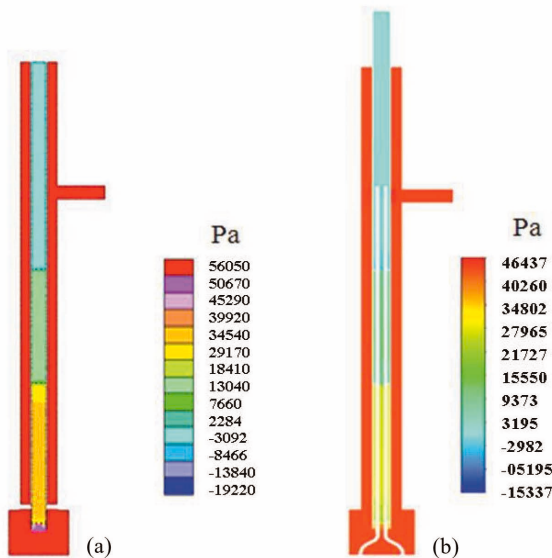

Fig. 7. Pressure Counters Results at a Plane Cross the Center Line of the Core (a) Star-CD, (b) CFX 
is its step profile with near uniform pressure in the subchannels and large pressure jumps across each of the spacers. In Figure 8, pressure overshoots occur in the spacer region, because the spacers undergo both sudden expansion and sudden contraction. As the flow area is suddenly contracted, with the area at the spacers' entrance being reduced by half, the pressure sharply decreases. The pressure is then recovered immediately, because the flow area suddenly expands, increasing twofold of it at the spacers' exit.

The first spacer undergoes a substantially larger pressure loss than the second and third spacer, respectively. However, at the first spacer, the pressure loss is a combined effect of acceleration and flow direction reversal due to the plenum flow entering the rod bundle. The Star-CD simulations result in a slightly higher pressure loss of 13 $\mathrm{kPa}$ than the CFX simulations, exhibiting a value near 11 $\mathrm{kPa}$ for the second and third spacers. Between spacers, a pressure loss of $\sim 2 \mathrm{kPa}$ pressure occurs, which is much less than the spacer pressure loss.

Comparison of the CFD results to the Rehme correlation reveals two main differences. First, the correlation does not discriminate between different spacers, and thust the first spacer with larger pressure loss is not distinguished from the other spacers exhibiting similar pressure loss. Second, the Rehme correlation, which was designed for specific spacer geometry, underpredicts the pressure loss $(\sim 7.5 \mathrm{kPa})$ of each spacer in the HELIOS mockup core. The simulated total pressure loss of the mockup core (Star-CD: $52 \mathrm{kPa}, \mathrm{CFX}: 46 \mathrm{kPa}$ ) compares very well with the experimental pressure loss $(52.5 \mathrm{kPa})$ for identical flow conditions. All other system code predictions obtained by the Rheme correlation for the spacers are substantially lower.

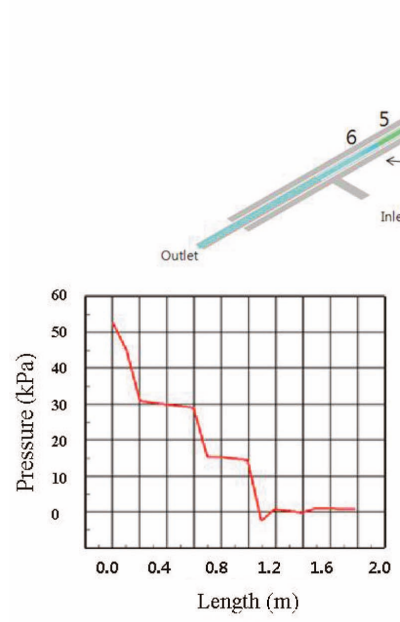

(a)
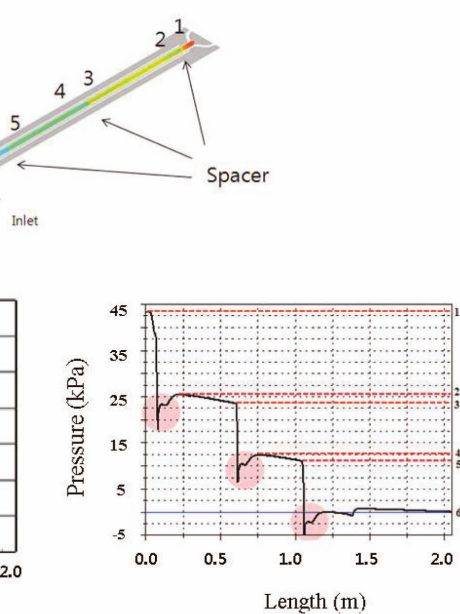

(b)
Fig. 8. Pressure along the Center Line Obtained for Tested Flow Rate, $13.57 \mathrm{~kg} / \mathrm{s}$ (a) Star-CD, (b) CFX

\section{CONCLUSIONS}

Based on system code modeling of the HELIOS loop in LACANES benchmarking phase I a substantial uncertainty with respect to pressure losses of the mockup core was noted. CFD simulations were performed for the mockup core, showing that spacer pressure losses are dominant. Consistent results of CFX and Star-CD were found. The results for each of the three spacers revealed substantially different pressure loss, since the flow is developing. Pressure loss ranges from 11 to $12 \mathrm{kPa}$ for the CFX simulation and from 13 to $14 \mathrm{kPa}$ for the Star$\mathrm{CD}$ simulation. The measured total pressure loss of the mockup core $(52.5 \mathrm{kPa})$ corresponds with the precalculated results of Star-CD (52 kPa) very well. CFX underpredicts the pressure loss by approximately $14 \%$. These small differences are related to different numerical meshes (polyhedral versus tetrahedral) and code specific wall treatment. Correlations used in LACANES benchmarking phase I for spacers were developed for specific spacer designs that differ from the present spacer design. CFD predictions provided more reliable results than any of these correlations.

In the complicated geometry of a reactor system, including the core and steam generator, correlations from handbooks have limitations regarding their applicability to prediction pressure losses due to the complexity of the geometry and the conditions of flow development. CFD simulations with suitable modeling options (sizes of mesh, turbulence model, wall treatment, etc.) should be adopted for hydraulic information used in system code simulations in order to ensure highly accurate predictions of pressure losses.

\section{ACKNOWLEDGMENT}

The work described in this paper was carried out under and supported by the FP6 EC Integrated Project EUROTRANS No. FI6W-CT-2004-516520. This work has also been supported by the Advanced Power Engineering Education Program of the KETEP under the Ministry of Knowledge and Economy, and in part by the Brain Korea 21 Program of the Ministry of Education, Science and Technology of Korea. Additional financial assistance of Sam Chang Company of Korea is deeply appreciated.

\section{REFERENCES}

[ 1 ] OECD/NEA, Benchmarking of thermal-hydraulic loop models for Lead-alloy cooled advanced nuclear energy systems (LACANES) - Task Guideline for Phase 1: Characterization of HELIOS, 2007.

[2] I.S. Hwang, et al., A Sustainable Regional Waste Transmutation System: P E A C E R, in ICAPP '06. 2006: Reno, NV, U.S.A.

[3 ] W.C. Nam, H.W. Lee, I.S. Hwang, Nucl. Eng. Des. 2007. 237(3): p. 316-324. 
[4] S.H. Jeong, Development of an Integral Test Loop, HELIOS and Investigation of Natural Circulation Ability for PEACER, in Nuclear Engineering Department, Seoul National University: Seoul, 2006.

[ 5 ] K.Rehme, "Widerstandsbeiwerte von Gitterabstandshaltern für Reaktorbrennelemente", Atomkernenergie, Vol. 15/2, pp. 127-130 (1970).

[6] N.E. Todreas, M.S.Kazimi, NUCLEAR SYSTEM I, Thermal Hydraulics Fundamentals, Hemisphere Publishing corporation, 1990, p. 382-389.

[7] B.E. Launder, D.B. Spalding, The numerical computation of turbulent flows, Comput, Methods Appl. Mech. Eng. 3 (1974) 269-289.

[8] K. Litfin, "EUROpean Research Programme for the TRANSmutation of High Level Nuclear Waste in an Accelerator Driven System", FI6W-CT2004-516520, Workpackage $\mathrm{N}^{\circ}$ : W 4.5, .Identification $\mathrm{N}^{\circ}$ : D 4.68.

[9] Batta, A. Class "Numerical Study on Flow Distribution and Turbulent Flow in XT-ADS Rod Bundle Water Experiment", The 8th International Topical Meeting on Nuclear ThermalHydraulics, Operation and Safety (NUTHOS-8), paper nr. N8P0232, Shanghai, China, October, 2010. 